\title{
Chemoreflex control of the cardiovascular system remains altered after recovery from low protein diet early in life
}

\author{
R.W.M. Sá ${ }^{1}$, G.S.M. Borges ${ }^{1}$, D.A. Chianca Jr., L.B. De Oliveira, L.M. Cardoso * \\ Federal University of Ouro Preto, Department of Biological Sciences/NUPEB Campus Universitário Morro do Cruzeiro, Ouro Preto, MG 35.400-000, Brazil
}

\section{A R T I C L E I N F O}

\section{Article history:}

Received 8 January 2014

Received in revised form 9 June 2014

Accepted 26 June 2014

\section{Keywords:}

Chemoreflex

Blood pressure

Protein restriction

Recovery from protein restriction

\begin{abstract}
A B S T R A C T
This study aimed to evaluate the cardiovascular component of the arterial chemoreflex in rats recovered from low protein diet. Male Fischer rats were randomly divided into control and recovered (R-PR) groups after weaning. R-PR rats were fed with low protein diet for 35 days and recovered under normal protein diet for 70 days. Control rats received normal protein diet for 105 days. Arterial chemoreflex was elicited by intravenous injection of KCN. Results showed that pressor response of the chemoreflex was increased in R-PR. Data suggest that protein restriction may alter cardiovascular response to chemical activation of the chemoreflex after recovery.
\end{abstract}

(C) 2014 Elsevier B.V. All rights reserved.
Cardiovascular diseases (CVD) are among the most common causes of mortality in the world. Among environmental factors contributing to CVD is the reduced protein intake. A reduction in protein diet leads to changes in cardiovascular homeostasis, affecting peripheral vascular resistance, renin secretion, renal hemodynamics and central neurotransmission of cardiovascular reflex pathways (Langley-Evans, 2001). The chemoreflex is an important regulator of blood pressure. Chemoreflex activation by cytotoxic chemicals or hypoxic hypoxia activates sympathoexcitatory and parasympathoexcitatory efferent drives promoting pressor and bradycardic responses, respectively. Chemoreflex control of the blood pressure is also known by its involvement with hypertension, especially because chronic chemoreflex activation could lead to sustained rise in mean arterial pressure (Fletcher, 2000). Data from literature show that cardiovascular component of chemoreflex is highly responsive in rats that underwent protein restriction early in life and may contribute to keep blood pressure within normal range in these animals (Penitente et al., 2007). The hypothesis that diet recovery could return cardiovascular response to chemoreflex activation back to normal never was properly addressed and tested. Therefore, this short study aimed to evaluate cardiovascular responses to chemoreflex chemical activation in rats recovered from low protein diet to address whether residual changes in the chemoreflex persisted after recovery.

Male Fischer rats were randomly divided into recovered (R-PR) and control groups. R-PR rats were fed with low protein (6\% protein, as casein) after weaning period ( 21 days after birth) for 35 days and

\footnotetext{
* Corresponding author at: Campus Universitário Morro do Cruzeiro, ICEB/NUPEB DECBI, Ouro Preto, MG 35.400-000, Brazil.

E-mail address: leomcardoso@gmail.com (L.M. Cardoso).

${ }^{1}$ Undergraduate students.
}

recovered under normal protein diet (20\% protein) for more 70 days. Control group received only normal protein diet for 105 days. Diets were made isocaloric by replacing protein content for carbohydrate (Tropia et al., 2001). Animals were kept in collective cages, under a 12-hour light/dark cycle and had free access to food and water. Room temperature was kept between 21 and $25{ }^{\circ} \mathrm{C}$. All procedures had the approval of the institutional animal care and use committee of the Federal University of Ouro Preto (protocol no. CEUA 2010/29) and were carried out according to EU Directive 2010/63/EU for animal experiments. Body weight and food intake were monitored during the whole treatment period. Under ketamine $(80 \mathrm{mg} / \mathrm{kg})$ plus xylazine $(7 \mathrm{mg} / \mathrm{kg}$ ) anesthesia, polyethylene catheters were inserted into the left femoral artery and vein for measurement of pulsatile arterial pressure (PAP) and drug administration, respectively. The free ends of the catheters were tunneled subcutaneously and exteriorized at the back of the neck. During experiments, arterial cannula was connected to a swivel and to a MLT0699 disposable blood pressure transducer (ADInstruments Pty Ltd, Australia). The analogical signal from the blood pressure transducer was preamplified by a ML 221 Bridge Amp (ADInstruments Pty Ltd, Australia) and digitized by an analog-todigital converter PowerLab 4/35 (ADInstruments Pty Ltd, Australia). Data were collected at a $1000 \mathrm{~Hz}$ sampling rate and a $20 \mathrm{mV}$ range digitizing window. Heart rate (HR) and mean arterial pressure (MAP) were derived on-line from pulsatile arterial pressure signal with LabChart 7.0 for Windows software. All experiments were performed in unanesthetized freely moving rats, approximately $48 \mathrm{~h}$ after catheter insertion surgery. Animals were housed in the experimentation room for $48 \mathrm{~h}$ before the experiments in order to allow full acclimation to experimental environment. Potassium cyanide (KCN) was obtained from Synth. Fresh made solutions (one for each dose) of KCN were 
prepared with PBS (phosphate buffered saline) $\mathrm{pH} 7.3$ so that the volume injected for each $\mathrm{KCN}$ dose ranged from 0.1 to $0.2 \mathrm{~mL}$. Baseline levels of mean arterial pressure (MAP) and heart rate (HR) were continuously recorded for $30 \mathrm{~min}$ after a 15 minute adaptation period. Arterial chemoreflex was elicited with intravenous (i.v.) injections of KCN according to previous studies which demonstrated that $\mathrm{KCN}$ is a potent activator of peripheral chemoreceptors in rats (Franchini and Krieger, 1993). Different doses of KCN (20, 40, 60, 80 e $160 \mu \mathrm{g} / \mathrm{kg}$ of body weight) were randomly injected i.v. at 5 minute interval. Data were expressed as mean \pm standard error of the mean (SEM). Baseline levels of MAP and HR were extracted from continuous recording (30 min) of each animal and averaged together to express group data. Cardiovascular responses to arterial chemoreflex activation were quantified as maximal change in MAP and $\mathrm{HR}$ from baseline as a result of $\mathrm{KCN}$ injections. Average changes in MAP and HR for each KCN dose in control and R-PR groups were analyzed by two way ANOVA followed by Bonferroni's pair wise test. Systolic interval (SI) and systolic blood pressure (SP) variability in the frequency domain were assessed by spectral analysis. A 30 minute period of continuous blood pressure recording was taken from the baseline recordings for spectral and spontaneous baroreflex analysis. Data from sequences of consecutive heartbeats were analyzed by the Cardioseries software v2.3. Both time series were resampled to $10 \mathrm{~Hz}$ by cubic spline interpolation and divided into contiguous segments of 512 values, which overlapped by 50\%. After Hanning windowing, the spectrum of each segment of either the SP or SI series was calculated using a fast Fourier transform (FFT) algorithm. Two oscillatory components were then compared between groups: low frequency band (LF; 0.20 to $0.75 \mathrm{~Hz}$ ) and high frequency band (HF; 0.75 to $3.00 \mathrm{~Hz}$ ). The peak of power density found in LF and HF bands from the spectra were averaged together in each group. Grouped data was compared using unpaired Student $t$-test. The relation LF/HF was also evaluated. The power density of HF and LF bands per se and the relationship between them has been widely used in the literature as a parameter to infer about sympathetic/parasympathetic relationship (autonomic balance) that governs heart rate (Goldstein et al., 2011). Spontaneous baroreflex sensitivity was computed using the sequence method, described by Parati et al (Parati et al., 1988) using the Cardioseries software v2.3. Briefly, the method is based on the identification of three or more consecutive beats in which progressive increases/decreases in systolic blood pressure are followed by progressive lengthening/shortening in systolic interval. Linear regressions of individual data are performed to assess slopes and all computed slopes were averaged together to obtain mean spontaneous baroreflex sensitivity (BRS). Data sets were considered statistically different when $\mathrm{p}$ value (probability of type I error) was smaller than 0.05 . All statistical analyses were performed by GraphPad Prism 6.02 for Windows.

After weaning, on the first day of treatment, control and R-PR groups had $62.4 \pm 3.4 \mathrm{~g}$ and $78.9 \pm 6.8 \mathrm{~g}$ respectively. After 35 days (end of the protein restriction period) control and R-PR weighed $184.3 \pm 7.3$ and $68.7 \pm 4.4 \mathrm{~g}$ respectively ( $\mathrm{p}<0.05$, two way ANOVA). At this point, R-PR rats had no body mass gain and weighed $62.7 \%$ less than control rats on average. Through this period, growth slope calculated by linear regression was smaller $(\mathrm{p}<0.0001)$ for R-PR group $(-0.2 \pm$ $0.11 \mathrm{~g} /$ day) compared to control group ( $3.5 \pm 0.14 \mathrm{~g} /$ day). After 5 days (40th day) of access to normal protein diet, R-PR rats started a rapid increase in body weight and body gain $(28.41 \pm 1.26 \mathrm{~g}$ for R-PR against $13.42 \pm 1.15 \mathrm{~g}$ for control on day 50 ), reaching body weight values close to control rats by day 85 . Growth slope for this period was higher $(\mathrm{p}<0.0001)$ for R-PR $(4.1 \pm 0.24 \mathrm{~g} /$ day $)$ compared to control $(1.9 \pm$ $0.20 \mathrm{~g} /$ day). At the end of the treatment period, control and R-PR groups had no significant differences on their body weights $(310 \pm 13 \mathrm{~g}$ for control and $291 \pm 16 \mathrm{~g}$ for R-PR) as well as in the body weight changes over time (growth slope $1.0 \pm 1.10 \mathrm{~g} /$ day for control and $1.9 \pm 1.46 \mathrm{~g} /$ day for $\mathrm{R}-\mathrm{PR}, \mathrm{p}=0.6383$ ). Average food intake was similar between groups throughout the entire experimental protocol. No significant differences were observed for MAP (109.3 $\pm 10 \mathrm{~mm} \mathrm{Hg}$ for control, $\mathrm{n}=7$ versus
$114.6 \pm 3.9 \mathrm{~mm}$ Hg for R-PR, $\mathrm{n}=5)$ and $\mathrm{HR}(332.5 \pm 16.3 \mathrm{bpm}$ for control versus $368.4 \pm 12.6$ bpm for R-PR) between groups. KCN produced transient pressor and bradycardic effects in a dose-response manner as previously described in the literature (Franchini and Krieger, 1993). The results summarized in Fig. 1 showed that pressor responses elicited by KCN injections were higher for the doses $60 \mu \mathrm{g} / \mathrm{kg}$ (23.5 \pm 3.6 $\Delta \mathrm{mm} \mathrm{Hg}$ for control versus $37.6 \pm 5.2 \Delta \mathrm{mm} \mathrm{Hg}$ for R-PR) and $160 \mu \mathrm{g} / \mathrm{kg}(36.9 \pm 2.8 \Delta \mathrm{mm} \mathrm{Hg}$ for control versus $52.5 \pm 4.2 \Delta \mathrm{mm} \mathrm{Hg}$ for R-PR) in R-PR rats compared to control rats. No significant differences were found by two way ANOVA for bradycardic responses between groups. Data from spectral analysis revealed no differences for LF and HF oscillatory components between control and R-PR rats. In addition, no difference was found between groups in the LF/HF ratio. Spontaneous baroreflex sensitivity was $44.12 \%$ higher in R-PR (0.49 \pm
A

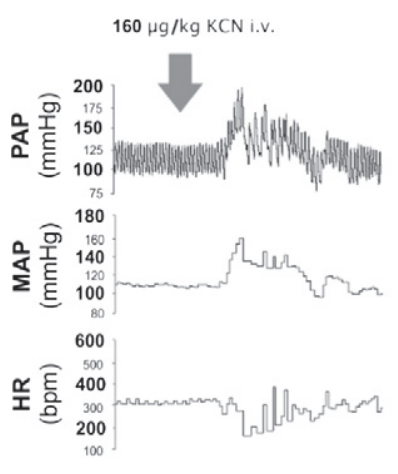

B
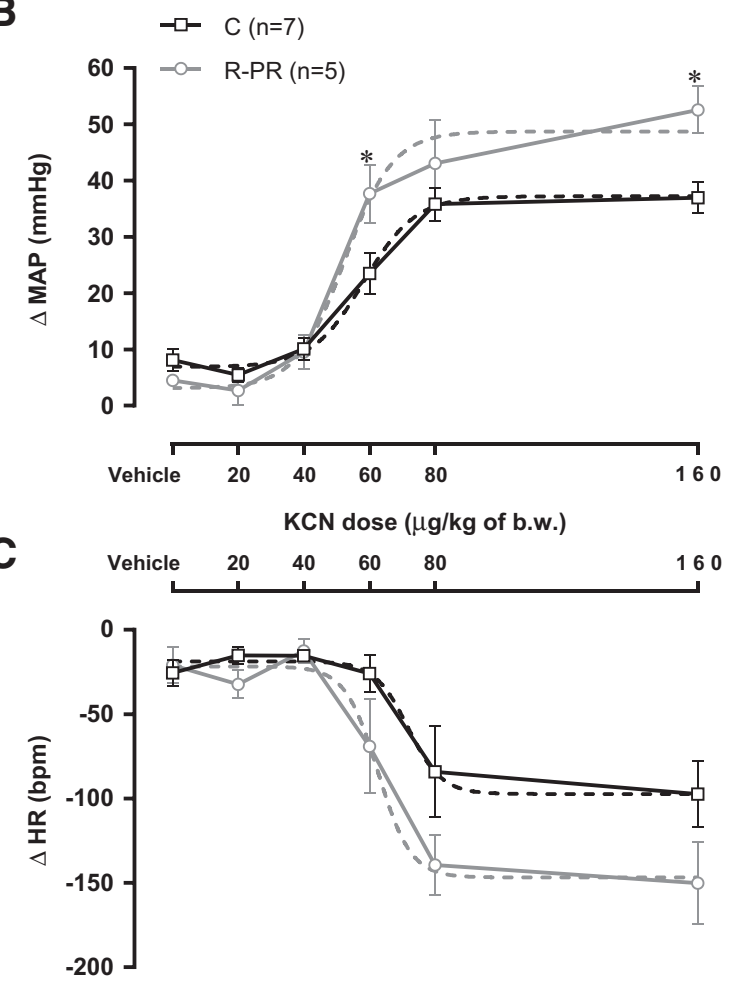

Fig. 1. (A) Traces of pulsatile, mean arterial pressure (PAP and MAP) and heart rate (HR) showing the profile of the cardiovascular responses elicited by intravenous (i.v.) injection of $\mathrm{KCN}(160 \mu \mathrm{g} / \mathrm{kg}$ of body weight) in a control and R-PR rat. Top arrows indicate the exact moment of the injection. Changes in MAP (B) and HR (C) elicited by activation of arterial chemoreflex with different doses of KCN. Dashed lines represent the best fit of the nonlinear regression ( 4 parameters) for each group. *Different from control; Two way ANOVA followed by Bonferroni's post-test. 
$0.04 \mathrm{~ms} / \mathrm{mm} \mathrm{Hg})$ than in control $(0.34 \pm 0.05 \mathrm{~ms} / \mathrm{mm} \mathrm{Hg})$ for R-PR group. Grouped data set is summarized in Table 1.

The goal of the present study was to determine whether recovery from a low protein diet restored cardiovascular responses to chemical activation of the chemoreflex. To establish the point of recovery, body weight was used as primary parameter to consider malnourished rats recovered from protein restriction without discrimination between fat and lean mass. Therefore, our proposed protocol (70 days of normal commercial diet) was capable to make statistically equal body mass of R-PR and control rats at the end of the protocol. The present results are not capable of assuring that changes in chemoreflex are residues of malnourish or secondary changes to diet switch during recovery period. However, rats from R-PR group ate about the same amount of regular diet and weighted about the same by the period they were experimented. Data also showed that mean arterial pressure and heart rate were within normal range after the recovery protocol what could suggest full recovery of regulatory mechanism related to blood pressure control. However, cardiovascular component of the arterial chemoreflex as well as spontaneous baroreflex gain were increased in R-RP rats compared to control. Previous data reported by Penitente and cols. showed increased responses to chemoreflex activation for both pressor and bradycardic components (Penitente et al., 2007). Our data indicate that pressor response elicited by arterial chemoreflex activation remained increased in R-PR rats, suggesting that the recovery protocol was not capable of restoring chemoreflex responsiveness back to normal in these rats. According to previous data in the literature, pressor component of the chemoreflex is dependent on sympathetic activation (Franchini and Krieger, 1993) suggesting that sympathoexcitation due to chemoreflex activation in RPR rats may be increased. This increased response could result from intrinsic chemoreceptor function, which may be more sensitive to KCN or adaptive changes in sympathetic pathways activated by chemoreflex. It has been reported in the literature that electrolytic lesions or inhibition of putative areas of the central nervous system involved with chemoreflex processing is capable of reducing arterial blood pressure in spontaneously hypertensive (SH) rats (Akemi et al., 2001) indicating that neuronal pathways activated by chemoreflex may be involved with maintenance of high blood pressure levels in such animals. It has also been postulated that increased respiratory-sympathetic coupling in early postnatal life may contribute to development of hypertension in SH rats (Simms et al., 2009) through changes in the central processing of cardiorespiratory modulation. However, our findings indicated that despite increased pressor response to arterial chemoreflex, curiously, blood pressure was not high in R-PR rats. In addition, spectral analysis data do not support increased sympathetic modulation of SI and SP. Therefore, we speculate that increased pressor response to chemoreflex stimulation may be involved with counter regulatory mechanisms to keep blood pressure within normal range along with increased baroreflex sensitivity. Among the possible outcomes, removal of peripheral chemoreflex may result in blood pressure increase, similar to previous results in malnourished rats (Penitente et al., 2007) or that increased pressor response could result from latent alterations corroborating with other factors to increase blood pressure levels late in life. Side studies, supporting the last hypothesis, showed that increased $\mathrm{NaCl}(2 \%)$ in the diet does not increase blood pressure by itself, but increase rostral ventrolateral medulla (RVLM) neurons excitability and lead to hypertension when combined with angiotensin II infusion (King et al., 2007; Stocker et al., 2010; Collister et al., 2013). Therefore, further studies are required to clarify the outcome of chemoreflex removal and interaction with baroreflex.

Overall power spectral analysis data showed that baseline autonomic modulation of cardiovascular functions is similar in both groups. However, chemoreflex and spontaneous baroreflex findings showed that important reflex mechanisms of cardiovascular control are changed after recovery. The meaning of these changes requires further investigations to be fully understood. However, clinical evidences suggest that nutritional challenges may produce outlasting alterations in cardiovascular regulation and autonomic control of the blood pressure may be an important target for those alterations. Studies revealed that individuals exposed to undernutrition early in life present higher probability of hypertension in adult life, even after recovered from that condition (Sawaya et al., 2005). Despite the fact that R-PR rats had no increase in blood pressure during the timeframe of this study, we cannot rule out the possibility of these animals to develop higher probability of hypertension. Enhanced peripheral chemoreflex regulation of the sympathetic drive seem to be involved with hypertension and may contribute to the development of high blood pressure levels late in life. Abdala et al. demonstrated that carotid body denervation reduces blood pressure in the developing spontaneously hypertensive rat (SHR) (Abdala et al., 2012). In addition, removal of carotid body inputs have been proposed as therapy for hypertension and can improve autonomic balance in heart failure (McBryde et al., 2013; Marcus et al., 2014) supporting the idea that increased sympathetic drive may involve chronic peripheral chemoreflex activation. The results of this study set the idea that, after access to normal protein diet, even though the early protein restricted rats gain mass and presented at the 105th day the same weight of control rats, pressor response of the peripheral chemoreflex remained altered. These results open the possibility for future investigations about the meaning of these changes in long-term control of blood pressure.

\section{Acknowledgments}

The authors thank the grants from Conselho Nacional de Desenvolvimento Científico e Tecnológico (CNPq grant no. 472254/ 2008-1), Fundação de Amparo à Pesquisa do Estado de Minas Gerais (FAPEMIG grant no. APQ-04161-10) and from the institutional programs of scientific initiation (PIBIC, PROBIC and PIP).

\section{References}

Abdala, A., McBryde, F., Marina, N., Hendy, E., Engelman, Z., Fudim, M., Sobotka, P. Gourine, A., Paton, J., 2012. Hypertension is critically dependent on the carotid body input in the spontaneously hypertensive rat. J. Physiol. 590, 4269-4277.

Table 1

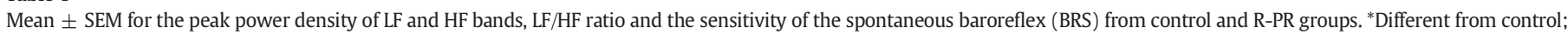
Student $t$-test, $\mathrm{p}<0.05$.

\begin{tabular}{|c|c|c|c|c|}
\hline & Control $(\mathrm{n}=5)$ & & R-PR $(\mathrm{n}=8)$ & \\
\hline MAP & $109.31 \pm 3.1 \mathrm{~mm} \mathrm{Hg}$ & & $114.69 \pm 3.9 \mathrm{~mm} \mathrm{Hg}$ & \\
\hline HR & $332.54 \pm 16.3 \mathrm{bpm}$ & & $368.41 \pm 12.6 \mathrm{bpm}$ & \\
\hline \multirow[t]{2}{*}{ BRS } & $0.34 \pm 0.05 \mathrm{~ms} / \mathrm{mm} \mathrm{Hg}$ & & $0.49 \pm 0.04 \mathrm{~ms} / \mathrm{mm} \mathrm{Hg}^{*}$ & \\
\hline & SI & $\mathrm{SP}$ & SI & $\mathrm{SP}$ \\
\hline Low frequency (LF) & $0.15 \pm 0.04 \mathrm{~ms}^{2} / \mathrm{Hz}$ & $5.49 \pm 0.97 \mathrm{~mm} \mathrm{Hg}^{2} / \mathrm{Hz}$ & $0.28 \pm 0.08 \mathrm{~ms}^{2} / \mathrm{Hz}$ & $4.24 \pm 0.34 \mathrm{~mm} \mathrm{Hg}^{2} / \mathrm{Hz}$ \\
\hline High frequency (HF) & $0.50 \pm 0.19 \mathrm{~ms}^{2} / \mathrm{Hz}$ & $1.17 \pm 0.10 \mathrm{~mm} \mathrm{Hg}^{2} / \mathrm{Hz}$ & $0.55 \pm 0.13 \mathrm{~ms}^{2} / \mathrm{Hz}$ & $1.24 \pm 0.09 \mathrm{~mm} \mathrm{Hg}^{2} / \mathrm{Hz}$ \\
\hline $\mathrm{LF} / \mathrm{HF}$ & $0.50 \pm 0.10$ & & $0.50 \pm 0.02$ & \\
\hline
\end{tabular}

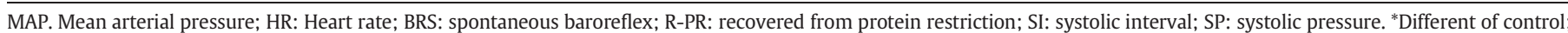
$\mathrm{p}<0.05$; Student's $t$-test. 
Akemi, S.M., Vanderlei, M.J., Ubriaco, L.O., Colombari, E., 2001. Lesions of the commissura nucleus of the solitary tract reduce arterial pressure in spontaneously hypertensive rats. Hypertension 38, 560-564.

Collister, J., Olson, M., Nahey, D., Vieira, A., Osborn, J., 2013. OVLT lesion decreases basal arterial pressure and the chronic hypertensive response to AngII in rats on a highsalt diet. Physiological reports 1.

Fletcher, E.C., 2000. Effect of episodic hypoxia on sympathetic activity and blood pressure Respir. Physiol. 119, 189-197.

Franchini, K.G., Krieger, E.M., 1993. Cardiovascular responses of conscious rats to carotid body chemoreceptor stimulation by intravenous KCN. J. Auton. Nerv. Syst. 42, 63-69.

Goldstein, D.S., Bentho, O., Park, M.Y., Sharabi, Y., 2011. Low-frequency power of heart rate variability is not a measure of cardiac sympathetic tone but may be a measure of modulation of cardiac autonomic outflows by baroreflexes. Exp. Physiol. 96, $1255-1261$.

King, A.J., Osborn, J.W., Fink, G.D., 2007. Splanchnic circulation is a critical neural target in angiotensin II salt hypertension in rats. Hypertension 50, 547-556.

Langley-Evans, S.C., 2001. Fetal programming of cardiovascular function through exposure to maternal undernutrition. Proc. Nutr. Soc. 60, 505-513.

Marcus, N., Del Rio, R., Schultz, E., Xia, X.-H., Schultz, H., 2014. Carotid body denervation improves autonomic and cardiac function and attenuates disordered breathing in congestive heart failure. J. Physiol. 592, 391-408.
McBryde, F.D., Abdala, A.P., Hendy, E.B., Pijacka, W., Marvar, P., Moraes, D.J., Sobotka, P.A., Paton, J.F., 2013. The carotid body as a putative therapeutic target for the treatment of neurogenic hypertension. Nat. Commun. 4, 2395.

Parati, G., Di Rienzo, M., Bertinieri, G., Pomidossi, G., Casadei, R., Groppelli, A., Pedotti, A. Zanchetti, A., Mancia, G., 1988. Evaluation of the baroreceptor-heart rate reflex by 24-hour intra-arterial blood pressure monitoring in humans. Hypertension 12, 214-222.

Penitente, A.R. Fernandes, LG Cardoso, L.M., Silva, ME Pedrosa, M.L Silva, A.L., Haibara, A.S., Moraes, M.F., Chianca, D.A., 2007. Malnutrition enhances cardiovascular responses to chemoreflex activation in awake rats. Life Sci. 81, 609-614.

Sawaya, A.L., Sesso, R., Florencio, T.M., Fernandes, M.T., Martins, P.A., 2005. Association between chronic undernutrition and hypertension. Matern. Child Nutr. 1, 155-163.

Simms, A.E., Paton, J.F., Pickering, A.E., Allen, A.M., 2009. Amplified respiratorysympathetic coupling in the spontaneously hypertensive rat: does it contribute to hypertension? J. Physiol. 587, 597-610.

Stocker, S.D., Madden, C.J., Sved, A.F., 2010. Excess dietary salt intake alters the excitability of central sympathetic networks. Physiol. Behav. 100, 519-524.

Tropia, F.C., Cardoso, L.M., Pedrosa, M.L., Silva, M.E., Haibara, A.S., Moraes, M.F., Chianca Jr., D.A., 2001. Effects of low-protein diet on the baroreflex and Bezold-Jarisch reflex in conscious rats. Nutr. Neurosci. 4, 99-107. 